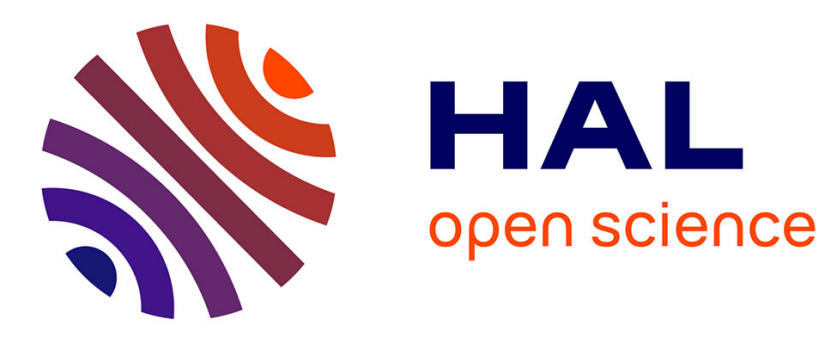

\title{
Les moteurs du développement territorial
}

André Torre

\section{To cite this version:}

André Torre. Les moteurs du développement territorial. Revue d'économie régionale et urbaine, 2018, Octobre (4), pp.711-736. 10.3917/reru.184.0711 . hal-02620408

\section{HAL Id: hal-02620408 \\ https: / hal.inrae.fr/hal-02620408}

Submitted on 25 May 2020

HAL is a multi-disciplinary open access archive for the deposit and dissemination of scientific research documents, whether they are published or not. The documents may come from teaching and research institutions in France or abroad, or from public or private research centers.
L'archive ouverte pluridisciplinaire HAL, est destinée au dépôt et à la diffusion de documents scientifiques de niveau recherche, publiés ou non, émanant des établissements d'enseignement et de recherche français ou étrangers, des laboratoires publics ou privés. 


\title{
LES MOTEURS DU DÉVELOPPEMENT TERRITORIAL
} André Torre

\author{
Armand Colin | « Revue d’Économie Régionale \& Urbaine »
}

2018/4 Octobre | pages 711 à 736

ISSN 0180-7307

ISBN 9782200931926

Article disponible en ligne à l'adresse :

https://www.cairn.info/revue-d-economie-regionale-et-urbaine-2018-4-page-711.htm

\section{Pour citer cet article :}

André Torre, «Les moteurs du développement territorial », Revue d'Économie Régionale \& Urbaine 2018/4 (Octobre), p. 711-736.

DOI 10.3917/reru.184.0711

Distribution électronique Cairn.info pour Armand Colin.

(c) Armand Colin. Tous droits réservés pour tous pays.

La reproduction ou représentation de cet article, notamment par photocopie, n'est autorisée que dans les limites des conditions générales d'utilisation du site ou, le cas échéant, des conditions générales de la licence souscrite par votre établissement. Toute autre reproduction ou représentation, en tout ou partie, sous quelque forme et de quelque manière que ce soit, est interdite sauf accord préalable et écrit de l'éditeur, en dehors des cas prévus par la législation en vigueur en France. Il est précisé que son stockage dans une base de données est également interdit. 


\title{
Les moteurs du développement territorial
}

\section{The drivers of territorial development}

\author{
André TORRE \\ UMR SAD-APT \\ Université Paris Saclay, INRA - AgroParistech \\ torre@agroparistech.fr
}

Mots-clés : Développement territorial, gouvernance, production, innovations

Keywords : territorial development, governance, production, innovations

Codes JEL: O01, O18, O31, O35 


\section{Résumé}

Cet article propose une analyse des deux moteurs du développement territorial : les processus de production et de gouvernance. Il examine leurs caractéristiques, leur fonctionnement et le rôle qu'ils jouent dans la mise en place des mécanismes de développement. Les modalités de création des innovations territoriales de différentes natures (technologiques, organisationnelles, sociales et institutionnelles) sont également étudiées. Le texte commence par un bilan de littérature et d'expériences sur les notions de gouvernance et de production au niveau territorial, suivi par une analyse de leurs modalités de fabrication des innovations territoriales. Pour finir, les mécanismes du développement territorial sont décrits sous forme de schémas, en s'appuyant sur trois options possibles : la loyauté ou la coopération, la prise de parole ou la concurrence, l'exit spatial ou la délocalisation.

\section{Abstract}

This article copes with the two drivers of territorial development - namely the production and governance processes. It examines their characteristics, their functioning, and the role they play in the implementation of territorial development mechanisms. Their link with territorial innovations of various natures (technological, organizational, social and institutional) is also examined. The text starts with a survey of literature and experiences on the notions of governance and production at the territorial level, followed by an analysis of the black box of fabrication of territorial innovation. To finish, the mechanisms of territorial development are described, based on three possible options of (non) development: loyalty or cooperation, voice or competition, spatial exit or relocation. 
Le processus de Destruction Créatrice représente la donnée fondamentale du capitalisme. $C^{\prime}$ est en elle que consiste, en dernière analyse, le capitalisme et toute entreprise capitaliste doit, bon gré mal gré, s'y adapter.

Joseph Schumpeter, Capitalisme, Socialisme et Démocratie. Petite bibliothèque Payot, 1942

Je n'avais, en ce temps-là, aucune idée du rôle considérable et d'une incontestable utilité que joue le bla-bla-bla dans la vie sociale des grands peuples vivant dans un état de liberté démocratique.

Winston Churchill, Mes jeunes années. Texto, Éditions Tallandier, 2007

\section{- 1 - \\ Introduction}

Dans un article un peu oublié aujourd'hui, Walter ISARD, le père de la Science Régionale, décrit le lien de l'homme au territoire à partir de la parabole d'un cosmonaute revenant de l'espace et rentrant sur terre (ISARD, 1956). A mesure que son vaisseau se rapproche de la surface de la planète, notre explorateur observe des paysages sans cesse plus détaillés, et passe d'un point de vue très global à l'observation des composantes des territoires. Il parcourt ainsi la gamme des échelles spatiales et économiques, des macro-systèmes aux processus de développement territorial, pour se trouver finalement, avant l'atterrissage, en mesure d'observer aussi bien l'activité et la vie quotidienne des populations que les mobilités des acteurs ou les réseaux qui les attachent ou les relient.

Cette fable illustre l'importance de la dimension territoriale, avec la diversité des parties prenantes locales, le rôle essentiel des questions d'occupation des sols et la prise en compte des processus sociaux et économiques dans les interactions entre personnes, entreprises ou institutions. Elle pose également l'ensemble des enjeux du développement territorial, de l'intérêt de se pencher sur les caractéristiques mêmes de la vie des territoires à la nécessité de comprendre leurs modalités de fonctionnement et de décrire les comportements et préoccupations des acteurs, au plus près du terrain.

Dans un précédent travail (TORRE, 2015), j'ai montré que la problématique du développement territorial, de retour dans les discours des chercheurs comme des politiques, se nourrit du lien concret au sol et de l'implication des différentes parties prenantes, mais également de la prise en considération des désirs et attentes des populations, toujours plus attentives et éduquées. A rebours du caractère macro ou top-down souvent inapproprié des interventions et incitations publiques, elle repose sur la légitimité des processus de gouvernance territoriale, qui impliquent les acteurs locaux dans la définition des enjeux et chemins de développement pour le futur. Cette évolution, qui signe la montée des attentes et de la participation des parties 
prenantes des territoires, se confronte aujourd'hui aux politiques généralistes vouées $\mathrm{au}$ «big is beautiful » et à la recherche d'économies par la suppression des échelons de démocratie représentative.

Mais cette préoccupation pour l'action publique et les idiosyncrasies territoriales ne doit pas conduire à négliger l'autre dimension des processus de développement. La production de biens et services, ainsi que la création, la multiplication et l'utilisation des ressources offertes par les territoires constituent en effet la seconde finalité du développement territorial. La mise en œuvre des chemins de développement ne peut reposer sur le seul transfert de richesses ; elle s'appuie avant tout sur la mobilisation, l'exploitation, et parfois l'exportation des ressources territoriales. Comme l'ont montré de nombreux chercheurs, le rôle joué par les systèmes de production, leurs ressources, leurs innovations, leurs réseaux et leurs interactions multiples, s'avère essentiel, sans négliger les relations entretenues avec d'autres territoires ou la place des consommateurs dans le choix des produits et de leurs caractéristiques.

Le développement territorial repose sur ses deux grands moteurs : la production et la gouvernance. C'est de leur genèse et de leur renouvellement permanent que naissent les processus de développement, déconstruisant et recréant des territoires sans cesse mouvants (LACOUR, 1996 ; MOINE, 2006), sous l'influence des dynamiques d'innovations technologiques, organisationnelles, sociales et institutionnelles, qu'elles soient endogènes ou provenant de l'extérieur (STORPER, 1997). Processus de gouvernance et de production donnent naissance à des innovations territoriales, qui accompagnent les mutations créatrices de nouveauté ou destructrices des anciens modèles de croissance et de gouvernement. Ainsi, le développement territorial peut marcher sur ses deux jambes, en intégrant à la fois l'accroissement des richesses et l'amélioration des caractéristiques socio-économiques des populations.

Le présent article propose une investigation des caractéristiques des deux moteurs du développement des territoires, de leur fonctionnement et du rôle qu'ils jouent dans les mécanismes de développement. Il commence par un bilan de littérature et de retours d'expériences sur les processus de production et de gouvernance, et se poursuit par une analyse de leurs modalités de fabrication des innovations territoriales. Pour finir, les mécanismes du développement territorial sont décrits sous forme de schémas, en s'appuyant sur trois options possibles : la loyauté ou la coopération, la prise de parole ou la concurrence, ainsi que l'exit spatial ou la délocalisation. Le lien avec les innovations territoriales de différentes natures (technologiques, organisationnelles, sociales et institutionnelles) est considéré tout au long du texte. 


\section{- 2 - \\ Le développement territorial : une question de gouvernance et de production}

L'évolution parallèle des analyses de la production et de la gouvernance, des injonctions macro aux dimensions locales, interroge leur rapport au développement des territoires. Les problématiques de développement reposent souvent sur l'intuition fondatrice de SCHUMPETER (1911), pour qui l'innovation permet de briser la routine des processus de production et de donner naissance à des phénomènes de destruction créatrice, avec l'apparition de nouveaux produits ou méthodes qui déclassent les anciens modes de faire et de penser. Elle est reprise et amplifiée par les déclinaisons territoriales, qui mettent l'accent sur la dimension technologique, alors que l'émergence des approches de la gouvernance territoriale s'effectue avant tout par la contestation des conceptions hiérarchiques du gouvernement et la reconnaissance de la multiplicité des parties prenantes des territoires.

\subsection{Le développement territorial porté par les processus de production}

Se développer, c'est d'abord produire : davantage, peut-être mieux, parfois moins... Des économistes classiques aux réflexions sur les pays émergents, la définition du développement est inséparable de celle de la production. La littérature sur les processus de développement des territoires ne fait pas exception, concentrée sur les activités productives et leur ancrage territorial (ZIMMERMANN, 2005). La production y est considérée comme le moteur principal du développement, avec une insistance sur deux dimensions centrales : l'innovation technologique et le caractère systémique des relations locales. Il s'agit ainsi avant tout d'une affaire de réseaux et de technologie.

Cette approche affirme le caractère situé et singulier du développement selon les territoires. Sa vision des systèmes locaux de production repose sur une double filiation. D'une part les systèmes nationaux d'innovation (NELSON, 1993), qui révèlent les idiosyncrasies des structures productives, institutionnelles et organisationnelles : les processus de production ne peuvent être identiques à Hong Kong et à Los Angeles en raison de particularismes locaux, de règles, de lois et de politiques économiques différentes. De l'autre, l'approche des pôles de croissance (PERROuX, 1964), qui favorise les grandes entreprises et industries susceptibles d'entraîner la croissance d'un territoire par le biais d'effets d'entraînement au sein de la structure économique locale.

L'analyse repose sur la dimension systémique des relations entre acteurs, au sein d'un territoire coproduit par leurs collaborations et projets communs. Interactions verticales et horizontales, corpus social homogène ou relations basées sur la répétition des échanges, l'important est la création d'un tissu local. Le développement territorial retrouve le focus infrarégional et la dimension productive du développement par le bas (STÖHR, 1986), mais l'élargit par les réseaux d'alliance et de coopération et la capacité à se renouveler et se transformer en réponse aux chocs exogènes. Il inspire 
ainsi de nombreuses politiques locales ou conduites par les pouvoirs publics, comme celles des pôles de compétitivité, des Spl ou des grappes technologiques en France.

\subsubsection{De l'importance de l'innovation technologique...}

Une importante littérature s'est attachée à la figure de l'entrepreneur (CASSON, 1982), innovateur audacieux, mais SCHUMPETER souligne également le caractère systémique des groupes de firmes innovantes et des grappes d'innovations. Pour les historiens des techniques (GILLES, 1978 ; ROSENBERG, 1982) une innovation n'apparaît jamais seule. L'idée d'une rupture issue du génie d'un homme est tempérée par l'existence de relations de collaboration et de coopération entre entreprises ou ingénieurs, et de complémentarités techniques : le développement du chemin de fer est dû à la découverte de la machine à vapeur mais aussi aux progrès réalisés dans la solidité des rails, le perfectionnement des aiguillages ou l'amélioration des techniques de transmission.

Remises à l'honneur par les philosophes des techniques et des organisations (SIMONDON, 1958), puis par les économistes évolutionnistes, ces approches montrent comment la transformation des connaissances et des inventions en innovations se traduit par des trajectoires technologiques (NELSON et WINTER, 1982), résultant des fortes opportunités offertes par certaines combinaisons techniques et économiques (la mécanisation plus facile de la fabrication du coton par rapport à la laine). Les innovations se diffusent, passent d'une entreprise ou d'un secteur à l'autre, puis deviennent incrémentales et routinières, produisant des effets de verrouillage. L'accumulation des connaissances, la stabilité institutionnelle et la forte inertie du modèle technologique dominant (Dosi, 1988) rendent difficile tout bouleversement, car la dépendance au sentier bloque les possibilités d'innovations non compatibles avec ce paradigme (DAVID, 1985). Ajoutons-y la transformation des produits de base en biens à fort contenu technologique, par une main-d'œuvre qualifiée ou des robots incorporant connaissances et innovations (LUNDVALL et MASKELL, 2000).

Les théoriciens de la transition (GEELS, 2002) expliquent la rupture de ce modèle routinier par le passage à un nouveau régime sociotechnique (AKRICH, 1994), fruit d'une ou plusieurs innovations radicales. Portées par des outsiders et protégées du système dominant par des subventions publiques ou des investissements stratégiques privés (VAN DE POEL, 2000), ces dernières incubent et se développent dans des niches qui permettent l'apprentissage de la technologie et la naissance d'une alternative sociotechnique de plus en plus stable et prometteuse. Quand apparaissent des limites technologiques ou des changements géopolitiques, culturels, démographiques dans le paysage d'ensemble, des "fenêtres d'opportunité "s'ouvrent, dans lesquelles s'engouffrent les innovations de niche les plus développées. Certaines vont percer, s'imposer, et donner naissance à leur tour à un nouveau régime sociotechnique.

\subsection{2. ... aux systèmes productifs locaux}

Ces approches en termes d'innovations systémiques, qui font le constat du caractère spatialement concentré des connaissances, trouvent un écho au niveau territorial avec l'analyse des systèmes productifs localisés, dont le modèle canonique s'est imposé en trois temps. 
Le mythe des districts industriels, déjà présents chez MARSHALL (1919), naît en Italie dans les années 1970 (BrusCO, 1982). Il s'agit du regroupement localisé de personnes et d'entreprises compétitives sur le marché mondial en dépit de leur (très) petite taille, comme la production textile du Prato, emblématique d'une forme de développement endogène à faible contenu technologique. Sociologues et économistes insistent sur les dynamiques sociales et le caractère très répandu de ces communautés de firmes historiquement liées par une division du travail au sein d'un même secteur (BECATTINI, 1990), qui échangent produits et main-d'œuvre dans un mélange de relations de concurrence et de coopération.

Rapidement, cette approche s'étend à d'autres types de regroupements localisés de producteurs, impliquant des laboratoires de R\&D et des firmes de tailles différentes, qui n'appartiennent pas toujours au même secteur (MARKUSEN, 1996). Les approches des milieux et des SPL (Systèmes Productifs Locaux) mettent ainsi l'accent sur un modèle plus générique, davantage axé sur les relations formelles et les échanges, dans lequel la production de connaissances est essentielle au développement territorial (CAMAGNI et MAILLAT, 2006). Les entreprises, liées par des relations de coopération, partagent des activités complémentaires au sein d'un ensemble spécialisé (COURLET, 2008), de plus en plus souvent marqué par une dimension technologique forte.

Enfin, PORTER $(1985,2003)$ impose, par sa souplesse analytique et son sens du marketing, le terme canonique de clusters. On retrouve l'idée d'un regroupement de firmes et de laboratoires travaillant dans des industries liées, au sein d'un environnement proche, et dont les interactions en termes de technologies et de savoir-faire permettent d'augmenter les performances, la compétitivité et le niveau d'innovation. Positionnée à l'origine du côté du management, l'approche dépasse rapidement ce champ, en dépit ou peut-être grâce à son flou analytique (VICENTE, 2016), avec une extension vers des systèmes moins axés sur les activités high tech ou au degré de performance plus faible (GIULIANI et BELL, 2005), puis comme un outil des politiques de développement, au niveau local ou national (OCDE, 2001).

\subsubsection{Approfondissements et extrapolations}

L'analyse des systèmes productifs localisés est prolongée par l'apparition de nouveaux avatars, comme les écosystèmes d'affaires, avec le débordement du périmètre de la firme par son insertion dans des réseaux de coévolution et de coopétition (BRANDENBURGER et NALEBUFF, 1996) caractérisés par la multiplication des acteurs (entreprises, laboratoires, centres divers) (MIRA-BONNARDEL et al., 2012). D'autres approches extrapolent davantage le modèle initial, en sortant des espaces urbains et de la technologie, comme les APL (Arrangements Productifs Locaux), qui renvoient au caractère parfois incomplet ou naissant des interactions productives dans les pays émergents (CASSIOLATO et al., 2003 ; JOYAL, 2008), ou les SyAL (Systèmes Agroalimentaires Localisés) (MUCHNIK et DE SAINTE MARIE, 2010), dont l'organisation résiliaire, entre producteurs et consommateurs, et la mise en commun de techniques et de savoir-faire locaux, s'avèrent essentielles dans les zones rurales.

La prise en compte des dimensions environnementales apporte une rupture plus radicale encore, avec la conscience que le développement se réalise aux dépens 
des ressources limitées de la planète. L'approche des Systèmes Socio-Écologiques (ANDERIES et al., 2004 ; OSTROM, 1990) approfondit les relations entre acteurs locaux et entités biophysiques et biologiques non-humaines, ainsi que les usages et la gestion collective des ressources et les exclusions qui en découlent. L'analyse des écosystèmes industriels va plus loin en intégrant le recyclage des outputs (DECOUZON et MAILLEFERT, 2012) et propose de substituer, à la succession d'opérations de transformation allant de l'utilisation des matières premières à la vente des produits, un modèle circulaire plus économe en ressources, qui réincorpore les déchets dans le cycle de production (JACOBSEN, 2006).

\subsection{Le rôle central des processus de gouvernance territoriale}

On sait que le développement ne concerne pas seulement l'augmentation, l'amélioration ou la diversification des productions; il recouvre bien d'autres dimensions, comme les changements mentaux et sociaux des populations, ainsi que les évolutions des structures institutionnelles (PERrouX, 1969 ; RIST, 2013). Pour décider de leur avenir et tenter de maitriser leur devenir, nations et territoires ont intérêt à prendre en main leur futur et à initier leurs propres projets de développement. Pour cette raison, le développement territorial ne peut s'appréhender indépendamment des processus de gouvernement et de gouvernance de la chose publique.

L'idée que le développement territorial doit prêter attention aux structures et modalités de gouvernance de l'action publique comme aux volontés des populations a mis du temps à s'affirmer, pour deux raisons. 1) Une vision du développement en termes de seules structures productives ou de degrés d'innovation, avec un intérêt marqué pour la compétitivité des territoires, horizon indépassable des politiques publiques locales ou déconcentrées. 2) Des approches du développement par le bas qui s'appuient souvent sur le mythe d'une organisation spontanée ou " naturelle » des acteurs locaux, s'exprimant ou agissant en dehors des problématiques organisationnelles ou de structuration des opinions (LAFONTAINE, 2005).

Paradoxalement, la remise en question est venue des débats sur les questions de gouvernance de l'entreprise, en particulier les modalités d'organisation interne des firmes et les formes d'externalisation des activités (MÉNARD, 1997). Ces préoccupations ont rapidement débordé sur le niveau territorial, avec le souci de gestion des sous-traitants et fournisseurs d'un même écosystème, puis la considération des modalités de gouvernance des systèmes locaux. Le succès de certaines technopoles ou zones d'activités high tech (Sophia Antipolis) renvoie logiquement à leurs modes de gouvernance. Il ne repose pas seulement sur la compétitivité des entreprises ou la qualité de leur mise en réseau, mais également sur la capacité des instances locales à manager et piloter des systèmes socio-économiques complexes et à faire respecter des règles admises par l'ensemble des participants. Ne dit-on pas souvent que c'est la vertu principale des pôles de compétitivité (GRANDCLEMENT, 2016) ? 


\subsubsection{De l'émergence des problématiques de gouvernance...}

Gouverner. Le mot peut sembler fort et réservé à un niveau élevé d'action des Etats et des nations. Pourtant, c'est tout simplement prendre des décisions, arbitrer des oppositions et des conflits, gérer des modes et des processus de production, et contribuer à la régulation des activités économiques et sociales (quand c'est possible).

L'idée de gouvernement a longtemps dominé, hiérarchique, descendante et contraignante, avec ses lois et ses politiques publiques. Puis, progressivement, a émergé le concept de gouvernance, parfois polysémique et flou, qui désigne des formes plus souples de pouvoir, reposant sur la coordination d'acteurs, de groupes sociaux et d'institutions en vue d'atteindre des objectifs communs (LE GALÉs, 2014). Cette évolution, qui s'inscrit dans un contexte d'innovations institutionnelles liées aux processus de décentralisation et de contractualisation, renvoie aux limites des politiques publiques, contestées par des populations aux attentes frustrées et impatientes de participer à leur élaboration. Elle fait également écho au mouvement de différentiation et d'autonomisation des composantes de la société, de multiplication des parties prenantes (PASQUIER et al., 2007) et à l'affirmation d'exigences de démocratie, au-delà de la seule représentation élective.

La gouvernance possède un versant normatif, avec ses préceptes et méthodes : la "bonne gouvernance ", prônée par la Banque Mondiale ou le FMI, propose des recettes censées garantir la compétitivité des pays ou des territoires. Mais le terme fait également référence à l'expérimentation de nouvelles modalités d'action publique et de participation des acteurs aux décisions, en rupture avec l'approche du gouvernement, dont l'organisation pyramidale est remise en question au profit de formes plus souples et proches des hommes et des organisations. Les réseaux d'acteurs économiques et sociaux voient ainsi leurs volontés et capacités d'expertise et d'innovation prises en considération dans la "fabrique » des politiques et de l'action publique (KOOIMAN, 2000) : intégration des partenariats public-privé à la définition des objectifs de développement (WeTteNHAL, 2003), participation d'organisations diverses (associations, entreprises, ONG...) à l'élaboration des lois, règles et réglementations (PIERRE, 2000), dispositifs facilitant la participation de parties prenantes aux processus de décision. La question de la gouvernance devient une préoccupation majeure des responsables politiques et cristallise les réflexions sur les questions d'interactions, d'action collective et de participation, d'intérêt général et de concertation.

\subsection{2. ... à la gouvernance territoriale}

Comme pour les processus de production, la focale, d'abord très globale, s'est déplacée vers les territoires, avec l'intégration des acteurs et de leurs problématiques de proximité dans la définition des objectifs et la mise en ouvre des décisions publiques (SUBRA, 2016). À l'intérêt pour les caractéristiques territoriales a répondu l'évolution des politiques - des équilibres macro au principe de subsidiarité - puis la montée de l'action publique (COMMAILLE, 2014). Les modèles de rapports au pouvoir central se sont multipliés, dans leurs dimensions institutionnelles, territoriales, d'identification, politiques... (PASQUIER, 2012), en intégrant progressivement les 
niveaux économiques et sociaux comme les fonctions régaliennes (LASCOUMES et Le GALES, 2007).

La tension des territoires avec un Etat gouvernant à distance par des dispositifs publics et des instruments (EPSTEIN, 2005) a alimenté la réflexion et le développement des problématiques de gouvernance territoriale. Elle repose sur l'idée que les différentes parties prenantes des territoires contribuent ensemble à leur pilotage, qu'il s'agisse des pouvoirs publics, des collectivités, des entreprises ou de différents groupes d'habitants (LELOUP et al., 2005). Longtemps tournées vers la contestation de la décision publique, les associations deviennent alors des parties prenantes de la discussion publique et de l'élaboration concertée (LASCOUMES, 1995), ainsi que de la mise en place de protocoles de négociation des normes au niveau local.

Les mécanismes de gouvernance territoriale sont à l'origine de changements de toutes natures dans les modes d'organisation et de fonctionnement des acteurs. Jamais stabilisés, ils suscitent débats et controverses, mais le consensus s'établit autour du caractère central de la participation des parties prenantes aux débats ou à la décision concernant l'avenir des territoires. La concertation permet de construire des objets communs pouvant faciliter une prise de décisions conjointes, présentes ou futures, en tenant compte des différentes contraintes et visions (TOUZARD, 2006). Créer les conditions de coopération, par exemple autour de la conception collective d'un projet ou de la planification des usages d'une ressource ou d'un espace (BEURET et CADORET, 2010), contribue à l'élaboration de projets partagés, sur la base de relations de coordinations plus ou moins équilibrées et hiérarchisées.

Enfin, le territoire est le lieu d'application par excellence de la gouvernance multi-niveaux (HOOGHE et MARKS 2001 ; BACHE et FLINDERS, 2004), dont les échelons territoriaux renvoient au fait que les décisions dépendent non seulement des directives et prescriptions des pouvoirs publics, mais également de Bruxelles, des services déconcentrés de l'Etat et des collectivités territoriales. Au-delà de la déclinaison des lois, règlements, politiques publiques ou instruments financiers, la gouvernance des territoires s'appuie en effet sur des instruments d'aménagement, qui déterminent l'occupation de l'espace, comme les Plans locaux d'urbanisme (PLUs) ou les Schémas de Cohérence Territoriale (SCoTs)... ainsi que sur des outils de gestion, comme les Communautés de Communes et d'Agglomérations, ou les Syndicats de bassins versants. Ils constituent autant d'outils dans les mains des acteurs.

\section{- 3 -}

\section{À la source des innovations territoriales}

Il convient de montrer comment les processus de production et de gouvernance que nous venons de présenter donnent naissance, au niveau des territoires, aux innovations et aux chemins de développement. Pénétrer la boite noire du développement territorial en explorant le fonctionnement et les causalités majeures de ses deux moteurs permet d'en préciser ou d'en définir les éléments constitutifs et les principes 
de base, dont certains restent négligés ou relégués au second plan par les approches territoriales exposées plus haut. C'est ainsi que l'on peut comprendre, en particulier, comment l'innovation territoriale est fabriquée par les processus de gouvernance et de production.

\subsection{Au cœur des relations de production : des innovations de différentes natures}

Produits agricoles, énergie, minéraux et minerais occupent une place déterminante dans le développement économique comme dans la production des innovations technologiques (CATIN, 1995). Il s'agit d'abord d'utiliser les ressources primaires, afin de mettre en marche l'appareil productif et nourrir une force de travail efficace et performante. Mais leur exploitation effrénée peut conduire à des modèles de développement fortement déséquilibrés, aux bénéfices territoriaux incertains comme l'approche des staples (INNIS, 1930). Utilisant successivement différentes matières premières (WATKINS, 1977), elle conduit à l'enchainement de phases d'accélération et d'étapes de récession fortes, accompagnées de pics de création et de destruction du tissu entrepreneurial et des filières.

Au-delà des dotations factorielles, les territoires leaders dans la course à l'industrialisation font longtemps la course du développement en tête (KRUGMAN, 1991). L'exploitation des ressources agricoles et naturelles et leur transformation par des travailleurs / consommateurs réclamant une diversité toujours plus grande de produits engendrent des phénomènes cumulatifs, qui profitent aux économies les plus précoces dans le passage au secteur secondaire. Les autres territoires en sont souvent réduits à exporter leurs ressources ou leurs productions intermédiaires (KRUGMAN et VenABLES, 1995). Même la variable démographique leur est défavorable. Formé et éduqué, le capital humain s'avère un puissant ferment territorial, par son potentiel en matière d'apprentissage et de production de connaissances, si bien que la rareté des compétences et des savoir-faire provoque une incitation forte en termes d'agglomération des personnes et des activités (LUCAS, 1988).

Les ressources spécifiques jouent ainsi un rôle décisif en matière de développement territorial (GUMUCHIAN et PECQUEUR, 2007), qu'il s'agisse de Capital, de matières premières, de travail ou de connaissance. Minerais bruts, main d'œuvre non qualifiée, produits agricoles de base... constituent autant de ressources génériques, aisément redéployables au-delà de leur territoire d'origine, et posent maintenant la question de leur caractère recyclable ou réutilisable. Alors que des ressources spécifiques comme une compétence artisanale, un fromage de terroir, un paysage remarquable, ne peuvent se reproduire ailleurs, car leur spécificité est liée au lieu d'origine. En générant de l'innovation territoriale par leur consommation sur place (tourisme...) ou leur transport (produits d'origine, avec forte spécification...) elles contribuent aux processus de développement (CAMPAgne et PECQUeUr, 2014). 


\subsubsection{Retour sur les trajectoires d'innovation}

L'approche des trajectoires technologiques, convaincante en matière de genèse ou de diffusion des innovations, présente la limite de ne pas considérer la dimension sociale ou institutionnelle comme une source d'innovation et de la traiter comme un simple accompagnement de l'innovation technologique. L'analyse des niches et de leurs transformations en opportunités technologiques (GEELS, 2002) ne s'applique en effet 1) qu'à des innovations fortes, ou de rupture...;2) certes portées par un paradigme dénommé socio-technique, mais qui s'avère avant tout de nature technologique.

Or une part importante des innovations territoriales est clairement 1) modeste (ALTER, 2010) ou frugale ; 2) avant tout de nature sociale et organisationnelle ou institutionnelle. Du coup cette approche, bien que cohérente, révèle une validité limitée au niveau des territoires. En effet, les innovations technologiques, qui impactent les structures productives pour s'y diffuser et y être adoptées, loin d'en être originaires s'avèrent souvent importées de l'extérieur. Bien qu'essentielles au progrès technique et aux dynamiques de croissance de l'Économie dans son ensemble par leur fonction d'induction, leur rôle au niveau du développement des territoires se révèle de facto bien plus limité. En témoigne l'échec de nombreux systèmes locaux d'innovation, qui peinent à faire émerger un réseau de conception locale des innovations technologiques. Les lieux de production de la technologie restent extrêmement limités sur la planète : quelques clusters, dans quelques régions, au sein d'un très petit nombre de pays, excluant de facto l'essentiel des territoires

\subsubsection{La multiplication des formes d'innovations territoriales}

L'analyse des systèmes localisés de production identifie le principe industriel des processus de développement. Mais elle n'embrasse pas l'ensemble de la question de l'innovation. Essentiellement technologique, cette conception doit être relativisée. En effet, tout pousse à un élargissement.

Tout d'abord, les consommateurs industriels jouent un rôle moteur dans la définition des produits et dans l'amélioration de leurs caractéristiques, avant leur mise sur le marché. Une position qui contraste avec celles des innovateurs de rupture, soucieux avant tout des questions de propriété intellectuelle. Les lead users, « utilisateurs pilotes » ou compétents, entretiennent ainsi des liens étroits avec le monde industriel et les fabricants de produits finals, en permettant d'améliorer leurs créations et de de peaufiner les innovations technologiques, le plus souvent dans une logique de partage et d'échange (VON HIPPEL, 1988).

Par ailleurs, de nouvelles modalités d'interactions innovantes émergent dans les territoires. Parfois qualifiées de "micro clusters ", les plateformes de services intégrés se multiplient. Espaces de démonstration, de co-working, fab labs, living labs, pépinières, centres de ressources... représentent des moyens de se rencontrer, d'échanger, d'interagir, voire d'élaborer des projets ou de construire ensemble des objets techniques. Ces tiers lieux présentent deux originalités : leur caractère hybride entre travail et loisir, mélangeant professionnels, citoyens et utilisateurs (OLDENBURG, 1991), et l'interaction épisodique entre participants, où se fabriquent des actifs 
relationnels. Loin d'être toutes high tech ces innovations, qui mêlent savoirs savants et profanes, s'avèrent ainsi de nature essentiellement organisationnelle ou sociale.

Enfin, l'approche des systèmes de production néglige les innovations modestes, qui reposent souvent sur la valorisation ou la spécification des ressources par les acteurs locaux (TORRE, 2015). On pense aux innovations sociales comme les crèches ou épiceries solidaires, les banques alimentaires, les aides aux personnes handicapées ... ou aux innovations organisationnelles faisant appel à une modification des modes de production, comme les circuits courts, la vente directe, le Micro crédit... C'est aussi le cas du mouvement des communs, avec la gestion collective de certains espaces fonciers ou l'accès à des biens locaux. Loin d'être anecdotiques, ces activités représentent aujourd'hui un volume d'emplois important et un axe de développement majeur des territoires.

Ainsi, les innovations territoriales ne se limitent pas aux seules dimensions technologiques et aux innovations de rupture. Il importe d'intégrer les innovations sociales (KLEIN et al., 2014), institutionnelles (BUREAU et CORSANI, 2015) et organisationnelles, ainsi que les nouveautés plus modestes et incrémentales (LORETO et al., 2017), dans le processus de développement territorial. Cette approche permet de comprendre par quels mécanismes sont produites les innovations territoriales et aussi d'estimer leur impact en termes de développement des territoires.

\subsection{Le caractère innovant des relations de gouvernance}

Les processus de coopération et de négociation facilitent la production de projets communs et l'introduction de nouveautés dans les modes d'organisation des territoires. Une fois pris ce tournant citoyen, il importe toutefois de ne pas verser dans une vision purement collaborative et délibérative de la gouvernance territoriale, et d'admettre que son fonctionnement peut être difficile et heurté, car il repose sur des relations dissymétriques, des oppositions, du pouvoir. Dispositifs, instruments et structures de gouvernance jouent le rôle de facilitateurs des interactions et contribuent à sa mise en action par l'édiction de règles tangibles, d'outils et de mécanismes innovants. Mais des obstacles subsistent : c'est de l'expression des conflits et du dépassement de ces laboratoires de l'action publique que naissent une partie des innovations territoriales et des projets de développement.

\subsubsection{Des instruments et mécanismes innovants...}

Nous définissons la gouvernance territoriale comme un processus de coordination entre des parties prenantes ou des acteurs de différentes natures (productifs, associatifs, particuliers, pouvoirs publics ou collectivités territoriales), aux ressources asymétriques, réunis autour d'enjeux territorialisés et contribuant avec l'aide d'outils et de structures appropriés à l'élaboration, parfois concertée, parfois conflictuelle, de projets communs pour le développement des territoires (TORRE et TRAVERSAC, 2011). Cette vision pragmatique, qui acte la dimension citoyenne du développement territorial (CHIA et al., 2008), renvoie à la construction d'outils et de représentations partagées (LEROUX, 2006 ; BEURET, 2010) et de territoires lieux de projets communs (REY-VALETTE et al., 2014). Elle répond à quelques objectifs simples : 
- contribuer à l'élaboration ou favoriser la mise en œuvre des projets de développement ;

- faciliter la coordination entre les parties prenantes hétérogènes au sein des territoires ;

- éviter que certains acteurs ne quittent le territoire (processus de désertification ou d'abandon) ;

- empêcher les affrontements bloquants ;

- décider des chemins de développement.

La coordination entre parties prenantes de natures très diverses ne constitue pas une évidence, ni le résultat d'arrangements spontanés. Pour fonctionner ensemble, les acteurs des territoires doivent utiliser ou fabriquer des dispositifs hétérogènes composés de personnes, d'institutions, d'aménagements, mais aussi de discours, de règles et de lois... (FouCAULT, 1975). La mise en action d'éléments verbaux, d'outils, de modes de fonctionnement et de structures d'organisation permet de développer un langage commun nécessaire au travail et aux actions concertées et d'initier des projets partagés. De la répétition des interactions et des apprentissages nait alors la pérennité des processus de développement territorial.

Outils et instruments occupent ainsi une place centrale dans les dispositifs de gouvernance, qu'il s'agisse de systèmes sociaux-économiques complexes, de structures de gouvernance, d'instruments techniques comme des textes, des documents de programmation, des contrats ou des chartes. Structurant les comportements des acteurs, qui occupent beaucoup de temps à les construire ou les approprier, ils font l'objet de batailles de pouvoir en termes de représentation (VITRY et CHIA, 2016). Leurs dimensions techniques et leurs principes de gestion, qui facilitent les interactions, cristallisent les rôles et positions au sein des organisations (HATCHUEL et WEIL 1992) au niveau territorial.

\subsection{2. ... aux obstacles et difficultés du processus de gouvernance}

Les dispositifs d'ingénierie de gouvernance en termes de modes de désignation de représentants ou de délibération jouent un rôle central dans la construction des chemins de développement. En effet, en dépit de la multiplication d'outils et d'instruments, la négociation territoriale ne va pas de soi et certains obstacles peuvent en entraver le déroulement, ou entacher les décisions de graves suspicions.

La réussite du processus de gouvernance dépend de deux conditions préalables : - L'acceptation des règles du jeu. Les parties prenantes peuvent refuser d'entrer dans le processus de définition d'un projet commun et quitter la partie. Le vote avec les pieds, c'est-à-dire le départ du territoire (TiEBOuT, 1956), s'avérant souvent impossible, le cas le plus courant est celui dans lequel un certain nombre d'acteurs, rétifs aux choix ou aux méthodes mises en œuvre pour y parvenir, abandonne la scène du développement territorial et choisit de ne pas s'exprimer ou d'agir en dehors des dispositifs de gouvernance, parfois en usant d'un pouvoir d'influence et de rapports de force ;

- La désignation des acteurs autour de la table, qui vont discuter et mettre en œuvre les projets de territoires et le processus de développement, puis en partager 
les bénéfices éventuels. L'assemblage suppose une phase arbitrale, de sélection et d'exclusion de certains acteurs (LEROUX, 2006), qui peuvent entrer en opposition. La taille nécessairement réduite de ces instances conduit à la désignation de groupes particuliers, représentés par des délégués portant leurs idées et leurs avis, et pose ainsi des questions de représentativité et de relais des opinions.

\subsubsection{Le conflit, mère de l'innovation territoriale}

L'arsenal de la gouvernance induit des réactions contrastées de la part des populations, qui tendent parfois à réagir et à s'opposer aux initiatives publiques ou privées. La contestation s'exprime par des recours aux tribunaux, des manifestations médiatiques ou des démonstrations de violence et se porte tout particulièrement sur les grands projets d'infrastructures (transport, production d'énergie, traitements des déchets) ainsi que sur les modes d'occupation de l'espace (Plus, permis de construire...) (TORRE et al., 2016). Ces conflits correspondent aux résistances à des décisions qui laissent insatisfaits certains acteurs locaux (DARLY et TORRE, 2013), ou à la remise en cause de la composition et de la représentativité des instances de décision. À côté de la coopération, ils représentent l'autre manière d'entrer en discussion sur les enjeux et les chemins du développement territorial, car leurs protagonistes peuvent espérer infléchir les décisions en prenant part au processus dont ils avaient été exclus (DOWDING et al., 2000), voire en imposant un changement ou un refus des modalités techniques.

Le conflit fait partie intégrante du processus de délibération territoriale. Certaines innovations - infrastructures, choix d'occupation des sols, structures de gouvernance - donnent naissance à des oppositions plus ou moins importantes selon les changements proposés. Au cours du conflit apparaissent à leur tour de nombreuses innovations, sociales et organisationnelles (constitution de nouveaux groupes d'acteurs), institutionnelles (nouvelles normes ou règlements) ou techniques (nouvelles solutions). Un processus d'apprentissage collectif, fait d'essais et d'erreurs, se met en place, qui permet de refuser, d'amender ou d'améliorer certaines propositions, de définir de nouveaux éléments de décision publique et de réintégrer des parties prenantes oubliées ou lésées dans des étapes antérieures. Il contribue également à faire renoncer l'adversaire ou à infléchir sa décision en incorporant une partie de ses arguments, tout en maintenant le dialogue, y compris durant les phases d'opposition les plus tendues.

\section{-4 - \\ Les mécanismes du développement territorial}

Abordons maintenant les mécanismes de mise en action des deux moteurs du développement des territoires et leurs modalités de production des innovations territoriales. Afin de décrire le processus de développement, nous reprenons et étendons l'analyse du triptyque exit, voice et loyalty de HiRSCHMAN (1970), qui propose une puissante explication des modalités de coordination au sein d'un collectif d'acteurs. Les liens aux situations et processus d'innovation territoriale 
s'expriment ici à travers trois options possibles : la loyauté ou la coopération, la prise de parole ou la concurrence, l'exit spatial ou la délocalisation.

\subsection{Les deux catégories $d$ 'Innovations territoriales}

Notre référence à l'innovation territoriale ne renvoie à aucun jugement de valeur. Il s'agit, au sens de SCHUMPETER, d'une nouveauté, importée ou produite par différentes composantes du tissu économique et social en réponse à des impacts exogènes ou à des initiatives locales. Jugée positive par les uns ou défavorable par d'autres (exemple d'une centrale nucléaire ou d'une prison), elle induit des changements dans les modes de fonctionnement antérieurs. Qu'il s'agisse d'innovations technologiques ou de rupture, ou de formes plus communes de nouveautés (au sens de LORETO et al., 2017) organisationnelles, sociales ou institutionnelles, elles reposent sur des inventions, ensuite validées par le marché, ou de nouveaux projets, qui recevront une validation de la société pour acquérir le statut d'innovations (Tableau 1, lecture verticale). Elles se décomposent également en deux catégories essentielles à la compréhension des processus de développement des territoires (lecture horizontale).

\section{Tableau 1 - Les formes d'innovations territoriales}

\begin{tabular}{|c|c|c|}
\hline & $\begin{array}{c}\text { Innovations } \\
\text { technologiques } \\
\text { et organisationnelles }\end{array}$ & $\begin{array}{c}\text { Innovations sociales } \\
\text { et institutionnelles }\end{array}$ \\
\hline Origine : inventions & $\begin{array}{c}\text { Origine : nouveaux } \\
\text { projets }\end{array}$ \\
\hline Coopératives/concertatives & $\begin{array}{c}\text { Produites par la } \\
\text { coopération }\end{array}$ & $\begin{array}{c}\text { Produites par la } \\
\text { concertation }\end{array}$ \\
\hline Concurrentielles/conflictuelles & $\begin{array}{c}\text { Produites par la } \\
\text { concurrence }\end{array}$ & Produites par le conflit \\
\hline
\end{tabular}

Les innovations coopératives ou concertatives résultent de l'acceptation par le marché ou par la société de propositions d'innovation ou de nouveautés venues de l'extérieur, ainsi que de la mise en ouvre de projets communs, de relations de coopération et parfois de confiance, entre différentes catégories de parties prenantes ou d'entreprises, qui produisent des formes nouvelles d'interactions et d'action collective. Il s'agit :

- d'innovations technologiques, avec les nouveaux processus de production au sein des filières...

- d'innovations organisationnelles, avec le développement de circuits courts ou de process de recyclage, de scops ou de chartes locales...

- d'innovations institutionnelles, avec la création de structures de gouvernance, Conseils d'arrondissement, Commission du Débat public, Schémas Régionaux de Cohérence Écologique...

- d'innovations sociales, avec les crèches partagées, les épiceries solidaires, la microfinance solidaire ou les laboratoires d'idées... 
Les innovations conflictuelles ou concurrentielles émergent en réaction à des initiatives prises par des acteurs publics ou privés, locaux ou extérieurs, qui conduisent à des processus de mise en concurrence de différentes solutions, ou à des réactions et oppositions à l'issue desquelles une solution est sélectionnée par la société ou le marché. Une bonne partie des innovations technologiques met en jeu des processus de concurrence entre firmes et/ou laboratoires, mais toutes les nouveautés ne sont pas bien reçues ou appropriées. Elles peuvent provoquer des résistances, voire des conflits, et être rejetées par tout ou partie des acteurs locaux. Ce constat, valide pour les innovations techniques (cf. le cas classique de l'introduction des métiers à tisser), l'est encore davantage pour les innovations sociales et institutionnelles, souvent sujettes à des oppositions irréductibles. Il s'agit :

- d'innovations technologiques, avec les nouveaux processus de production ou de recyclage...

- d'innovations organisationnelles, avec les nouveaux tours de tables ou les restructurations des groupes de pression ;

- d'innovations institutionnelles, avec les nouvelles modalités de débat public, l'organisation de groupes de négociation...

- d'innovations sociales, avec les changements de rapports de force entre groupes d'opposants.

\subsection{Fabrication des innovations territoriales par les processus de gouvernance}

La gouvernance des territoires ne se réduit pas aux formes de coopération et de constructions communes (TORRE et TRAVERSAC, 2011). Il s'agit plutôt d'une interaction entre des forces qui poussent à la coopération et d'autres au conflit. Les processus de développement territorial sont faits de phases de négociations, de collaboration ou d'apaisement, mais également de périodes beaucoup plus animées, ou conflictuelles, au cours desquelles certains groupes ou catégories d'acteurs s'opposent, parfois avec virulence, pour définir la marche à suivre et les options à retenir (MELÉ, 2013). Ils présentent ainsi deux faces complémentaires, qui contribuent à la production des innovations territoriales et dont l'importance réciproque varie selon les périodes et les situations (GLAZER et KONRAD, 2005).

Élaborée à l'origine pour rendre compte des réactions des consommateurs face à une détérioration de qualité de la production d'une entreprise, l'approche d'HiRSCHMAN peut être transposée à d'autres situations, comme celles du développement des territoires. L'extrapolation au cas des relations territoriales révèle que les acteurs peuvent développer, en réaction à une initiative initiale, trois grands types de comportements, qui donnent naissance à autant de chemins de (non) développement (TORRE et BEURET, 2012) ${ }^{1}$.

La loyauté consiste à accepter la décision prise et à " jouer le jeu » en silence ou à co-construire l'innovation territoriale de nature coopérative. C'est l'adhésion aux décisions en cours, par une participation aux dynamiques et projets, voire par l'absence d'opposition publique ou l'attente d'une sanction éventuelle par un passage ultérieur par les urnes. Elle correspond à l'acceptation sans opposition d'un projet 
de développement, ou à sa mise en ouvre à la suite d'un processus de concertation réussi, voire dans lequel les opposants renoncent et préfèrent se plier à la décision majoritaire ou des organisations les plus puissantes.

La prise de parole, ou voice, consiste à s'opposer, de manière légale ou non, à une décision et à la contester publiquement. Les conflits sont une expression du voice, quand tout ou partie de la population est insatisfaite des décisions ou des projets, se sent négligée dans les négociations ou non représentée dans les structures de gouvernance. Il peut prendre une expression individuelle, plutôt réservée aux petits conflits, ou collective, avec élargissement de l'espace de concernement et mobilisation contre des projets de taille importante ou impliquant une reconfiguration forte des modalités de gouvernance territoriale (DowDING et al., 2000). L'opposition est alors souvent dirigée vers les pouvoirs publics, dans l'objectif de remettre en question leurs décisions et d'influencer le processus de gouvernance (MARTINAIS, 2015).

L'exit revient à voter avec les pieds (ТІевоuт, 1956) ou à sortir du jeu, et en particulier du territoire. Il va sans dire que cette solution de non développement n'est pas toujours praticable; par exemple, pour des raisons de coûts ou de prix du foncier (il est difficile de revendre à bon prix des terrains pollués ou proches d'une source importante de nuisances) ou d'opportunités de relocalisation. Nous la qualifions d'exit spatial, patent dans des territoires touchés par la désertification, l'atonie ou l'isolement économique et institutionnel, comme certains espaces ruraux ou périphériques. Ou dans des zones de conflits si violents que toute régulation semble impossible et recommande l'exil. Dans de telles situations, les liens ont tendance à se déliter au niveau territorial, où à se révéler insuffisants pour contenir la montée des tensions.

La Figure 1, dans laquelle le territoire est représenté par un ovale, illustre le mode de production des innovations territoriales à partir de ces trois options. Les solutions, qui correspondent à autant d'idéaux-types de comportements des acteurs, sont conditionnées par les mécanismes coopératifs et conflictuels ou les processus d'exit spatial. Les nouveautés peuvent être endogènes ou importées de l'extérieur, se révéler modestes ou importantes ; les innovations seront qualifiées à l'issue du processus, en fonction de leur réception par la société. Les chemins de développement qui en découlent ne s'excluent pas mutuellement et peuvent cohabiter dans le temps (TORRE, 2015). Il arrive que certains prennent le dessus et impriment un « esprit local », plus ou moins collaboratif, atone ou conflictuel.

La situation de coopération fait reposer la dynamique des projets sur la conjonction des volontés des parties prenantes (concertation/loyauté), conduisant à la production d'innovations concertatives. Dans le cas des dynamiques d'opposition et de séparation (conflits/voice, exclusion) c'est l'absence d'adhésion générale à un projet qui domine, en multipliant les options contraires et les espoirs de reconfigurations. Les processus conflictuels font alors naitre des innovations conflictuelles et de nouveaux chemins de développement suite à la révision des plans initiaux des acteurs privés ou publics. Les mécanismes d'exclusion compartimentent des groupes 
particuliers et peuvent conduire à une ségrégation spatiale contribuant au morcellement des territoires. Enfin, l'impuissance à générer ou maintenir des solidarités et des échanges - fussent-ils conflictuels - peut provoquer le départ du territoire d'une partie des acteurs (exit spatial), et l'apparition de processus d'atonie et de déprise. Le non développement prend alors le dessus, sans production d'innovations.

\subsection{Fabrication des innovations territoriales par les processus de production}

Le même exercice peut être réalisé pour illustrer le rôle joué par les relations de production dans les processus de développement territorial, en étendant le tripode initial aux comportements productifs. La logique de base, identique, repose sur trois grandes figures. Les rapports de coopération entre acteurs prennent la forme du travail réalisé en commun, des alliances et réseaux. Aux relations de concurrence entre entreprises ou sur le marché du travail répondent les processus de délocalisation des firmes.

La coopération entre entreprises relève avant tout d'une stratégie de mise en commun ou d'échange de compétences et de connaissances, dans l'objectif d'un gain productif ou la fabrication de produits communs. Les formes possibles, qui vont du réseau d'échange technologique à l'entreprise conjointe ou à la coopérative, en passant par les accords et alliances de différents types, font avant tout appel à la recherche d'économies d'échelle ou à la réduction des coûts de transaction. La coopération s'appuie généralement sur la répétition des relations et sur une défiance réciproque et bien comprise, validée par des contrats et des accords formels, ou sur des relations tacites. Elle peut également naitre des relations de confiance entre acteurs appartenant à une même communauté par exemple.

Les rapports de concurrence, l'un des moteurs majeurs du capitalisme, ne sont pas toujours exacerbés au niveau territorial, où dominent souvent les situations d'oligopole et de monopole, excepté pour les activités de services et de commercialisation, où la compétition fait rage entre différentes enseignes ou commerçants. En revanche, les entreprises y combinent souvent relations de concurrence et de coopération, stratégies d'alliance ou d'opposition selon les fonctions concernées (R\&D, production, commercialisation...), comme souvent relevé au sein des systèmes localisés de production.

Les délocalisations représentent une sortie du territoire pouvant concerner l'ensemble des fonctions d'un établissement ou seulement une partie (transfert d'un volet de la production, d'une étape de fabrication, d'un process industriel ou d'un service) (MESSAOUDI, 2014). Longtemps limitées à des mouvements intra-nationaux, elles ont pris de l'ampleur avec la globalisation, en s'étendant à d'autres pays. Qu'il s'agisse d'une cessation complète d'activité ou d'allers-retours des produits engendrés par la décomposition internationale des processus productifs (JENNEQUIN et al., 2017), elles provoquent un déficit d'emplois net pour le territoire d'origine, en particulier quand elles touchent des bassins d'emplois déjà fragiles ou spécialisés, 


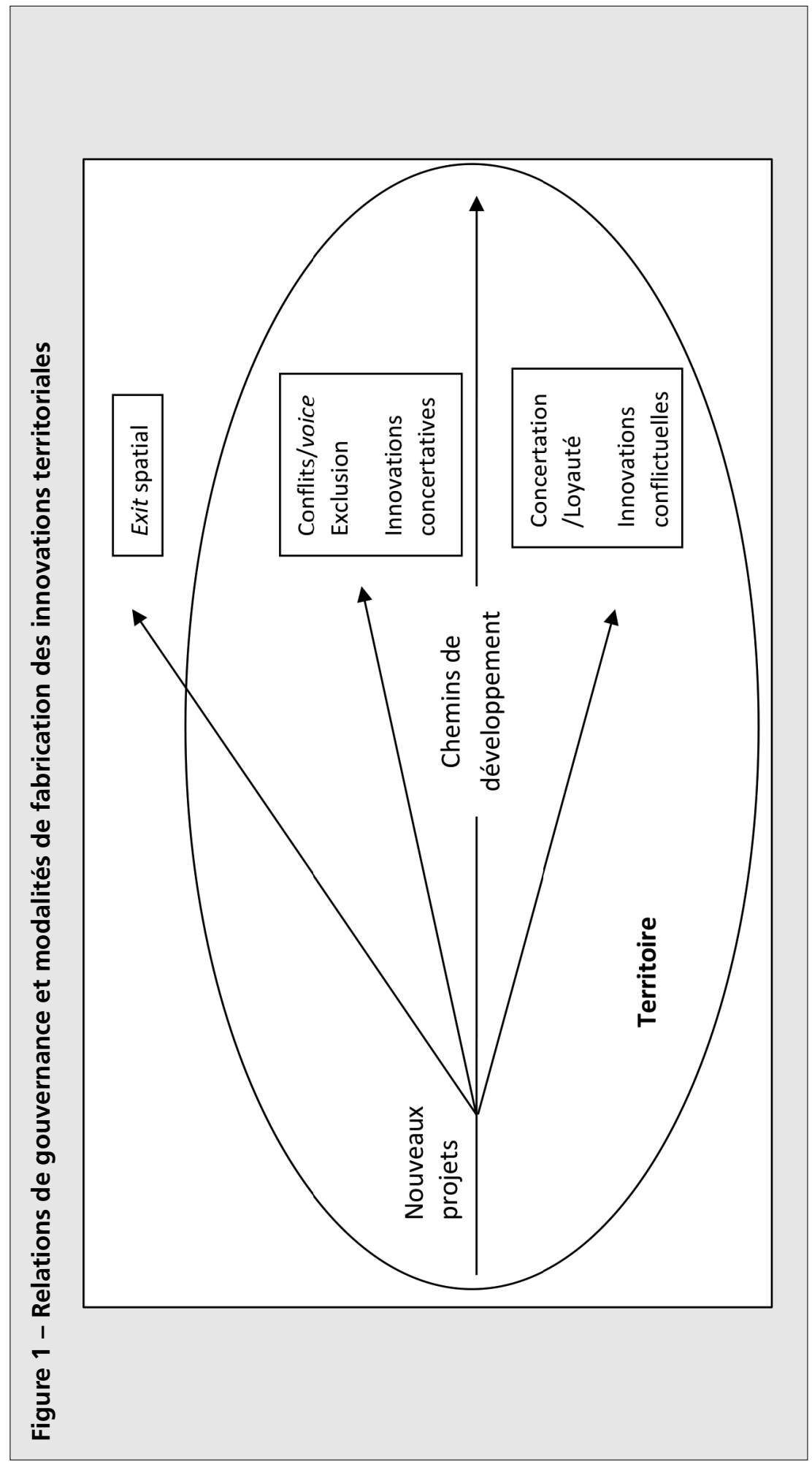


accentuant la perte de lien au territoire et la déconnexion des relations industrielles locales.

La Figure 2 illustre la mise en action de ces trois options dans des situations de production. Elle présente les différentes voies de développement possibles, ainsi que la production d'innovations technologiques et organisationnelles, qualifiées par le marché et résultant de processus internes ou importés de l'extérieur. Ici encore ces différents chemins ne sont pas exclusifs, voire même cohabitent comme le montre l'exemple de la coopétition. Toutefois, la dominante de l'une ou l'autre option va déterminer l'esprit d'un territoire, plutôt concurrentiel (une zone commerciale avec différentes enseignes), coopératif (une grappe d'entreprises), voire franchement déprimé (une zone industrielle soumise à des fermetures d'usines).

\section{- 5 - Conclusion}

CHuRCHILl avait raison ; la discussion, surtout quand elle prend une expression publique, est essentielle aux sociétés démocratiques et constitue la matrice des processus de gouvernance. Les échanges et la communication, avec les accords qu'ils construisent, les oppositions qu'ils portent et permettent de clarifier ou de résoudre, sont au fondement du fonctionnement des sociétés humaines. Les territoires ne font pas exception. Discuter, élaborer, contester, y compris par l'intermédiaire des nouveaux moyens de communication comme les réseaux sociaux, représente une part essentielle des processus de développement territorial, et permet d'exprimer la diversité des intentions humaines. C'est à ce prix que peut émerger et s'entretenir le processus de destruction créatrice, avec sa dynamique, ses ambiguïtés et ses contradictions, et que les innovations territoriales naissent des conflits et des coopérations.

L'autre moteur du développement des territoires, l'activité productive, repose sur les entreprises, les services privés et publics et les exploitations agricoles, qui exploitent et génèrent les ressources territoriales. Les innovations technologiques et organisationnelles, élaborées localement ou transférées et adaptées de l'extérieur, induisent des modifications des structures de production, en favorisant l'apparition de nouveaux produits, de nouvelles méthodes de fabrication ou de nouvelles entreprises, de nouveaux rapports économiques, rendant obsolètes les anciennes manières de faire et menaçant les emplois et structures existants. Les irréversibilités auxquelles elles donnent naissance signent l'importance de la dimension technique et contribuent à la réorganisation et à l'évolution des tissus socio-économiques locaux.

Le processus de développement des territoires nait ainsi de l'entrelacs incessant des dimensions productives et de gouvernance, du ronron et parfois du rugissement de ses deux moteurs. Ses dynamiques résultent de la conjonction de nombreux chemins de développement, avec leurs avancées rapides, leurs blocages, ou leurs retours 


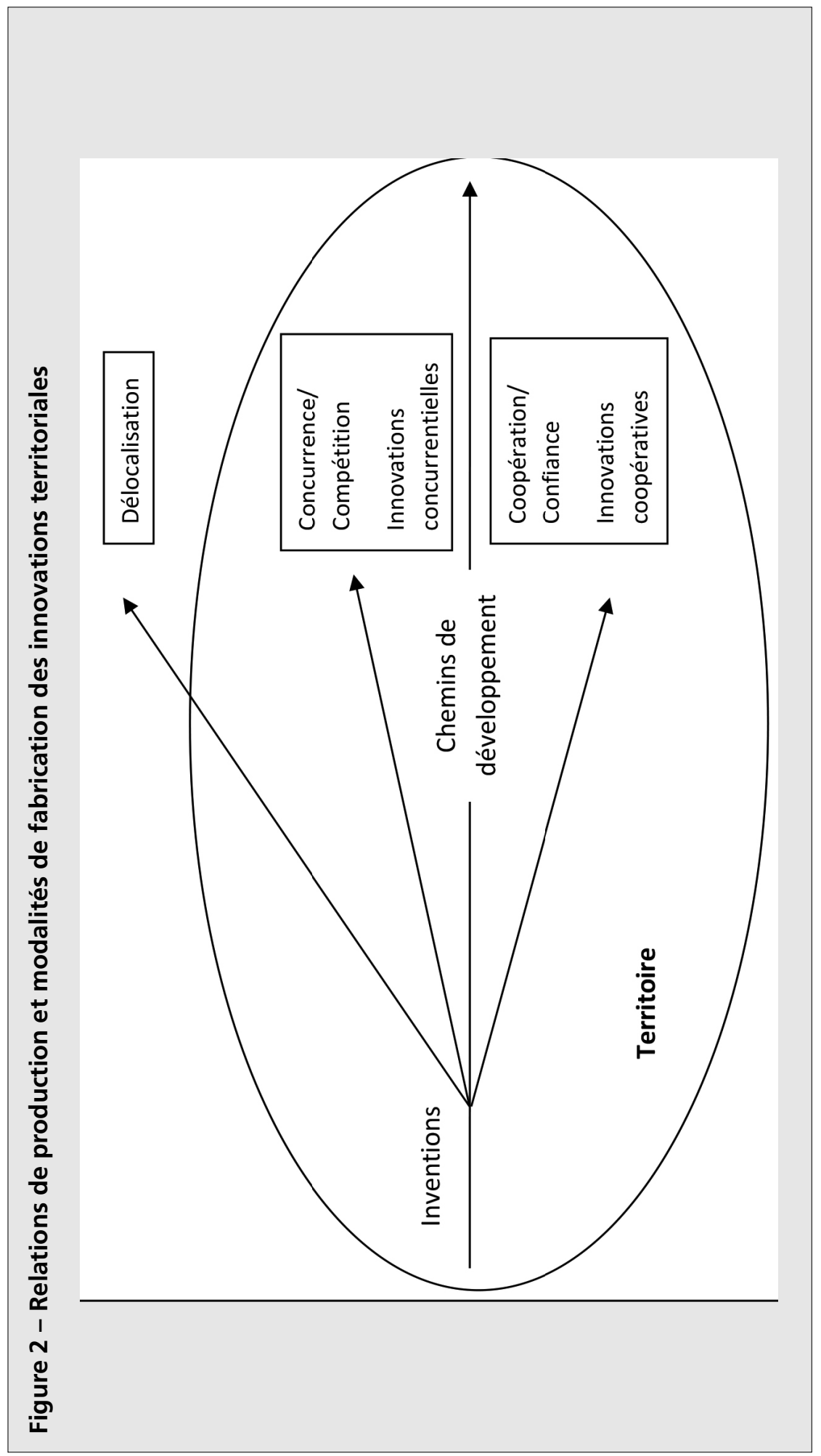


en arrière. Elles se révèlent propices à des mécanismes de destruction créatrice plus ou moins puissants, qui provoquent des changements économiques, des évolutions des mentalités, des bouleversements des structures institutionnelles et des reconfigurations des réseaux sociaux. La complexité des processus d'innovation impose le recours à des expérimentations locales qui, en cas de succès, pourront se diffuser ou se reproduire ailleurs. Reste à définir jusqu'où peuvent se projeter les dynamiques ainsi envisagées, aux dépends des ressources naturelles, de la biodiversité et de la disponibilité des sols, dans une période de changement climatique avérée.

\section{Remerciements}

L'auteur tient à remercier Claude LACOUR pour une relecture rigoureuse et affectueuse, Danielle LAFONTAINE pour la révélation de l'article de Walter ISARD, inspirant de nombreuses réflexions ultérieures, ainsi que trois rapporteurs anonymes pour leurs remarques aiguisées et déstabilisantes.

\section{Bibliographie}

AKRICH M (1994) Comment sortir de la dichotomie technique/société. Recherches 1 : 103-131.

ALTER N (2010) L'innovation ordinaire. Quadrige, PUF, Paris.

ANDERIES J-M, JANSSEN M-A, OSTROM E (2004) A Framework to analyze the Robustness of Social-ecological Systems from an Institutional Perspective. Ecology and Society 9(1), article 18 [en ligne] http://www. ecologyandsociety.org/vol9/iss1/art18/

Beccatini G (1990) The Marshallian District as a Socio-Economic Notion. In: Pyke F, BeCCATINI G, SENBENGERBER W (Eds) Industrial districts as Inter-Firms Co-operation in Italy. International Institute for Labour studies, Genève : 37-51.

BEURET J-E (2010) De la négociation conflictuelle à la négociation concertative : un point de passage transactionnel. Négociations $1: 45-60$.

Beuret J-E, CAdoret A (2010) Gérer ensemble les Territoires. Charles Léopold Mayer, Paris.

Brandenburger A-M, Nalebuff B-J (1996) Co-opetition. Doubleday, New York.

BRUSCO S (1982) The Emilian model: productive decentralisation and social integration. Cambridge Journal of Economics 6: 167-184.

BuREAU M-C, CORSANI A (2015) Les coopératives d'activité et d'emploi : pratiques d'innovation institutionnelle. Revue Française de Socio-Économie 15 (1) : 213-231.

CAmagni R, Maillat D (2006) Milieux innovateurs. Théories et politiques. Collectoin Anthropos, Economica, Paris.

CAmpagne P, PeCQueur B (2014) Le développement territorial. Une réponse émergente à la mondialisation. Charles Léopold Mayer, Paris.

CAssiolato J-E, Lastres H-M-M, MaCiel M-L (2003) Systems of Innovation and Development: Evidence from Brazil. Edward Elgar, Cheltenham.

CASSON M (1982) The Entrepreneur, An Economic Theory. Barnes and Noble. New York.

CATIN M (1995) Les mécanismes et les étapes de la croissance régionale. Région et développement 1 : 27-63. 
Chia E, Torre A, ReY-Valette H (2008) Vers une « technologie » de la gouvernance territoriale ! Plaidoyer pour un programme de recherche sur les instruments et dispositifs de la gouvernance des territoires. Norois 209 : 167-177.

COMmaille J (2014) Sociologie de l'action publique. In : BousSAGuet L, JACQUOt S, RaVINET P (dir.) Dictionnaire des Politiques Publiques. Presses de SciencesPo, Paris.

COURLet C (2008) L'Économie Territoriale. Presses Universitaires de Grenoble.

DARLY S, TORRE A (2013) Conflicts over farmland uses and the dynamics of "agri-urban" localities in the greater Paris region. Land Use Policy 33: 90 - 99.

DAVID P-A (1985) Clio and the Economics of QWERTY. The American Economic Review, Papers and Proceedings of the Meeting of the American Economic Association 75(2): 332-337.

DeCOuZON C, MAILLEFERT M (2012) Évaluer des projets d'écologie industrielle sur des parcs d'activité : des synergies au territoire. Géographie, Économie, Société 14 (4) : 411-434.

Dosı G (1988) Sources, Procedures, and Microeconomic. Effects of Innovation. Journal of Economic Literature, XXVI, Sept.: 1120-1171.

DOWDING K-J, JOHN P, MERGOUPIS T, VAN VuGT M (2000) Exit, voice and loyalty: Analytic and empirical developments. European Journal of Political Research 37: 469-495.

EPSTEIN R (2005) Gouverner à distance. Quand I'Etat se retire des territoires. Esprit 11 : 96-111.

FouCAult M (1975) Surveiller et punir. Gallimard, Paris.

GEELS F-W (2002) Technological transitions as evolutionary reconfiguration processes: a multi-level perspective and a case-study. Research Policy 31(8-9): 1257-1274.

GILLES B (1978) Histoire des techniques. La Pléiade, Gallimard, Paris.

Giuliani E, Bell M (2005) The micro-determinants of meso-level learning and innovation: evidence from a Chilean wine cluster. Research Policy 34(1): 47-68.

Glazer A, KOnRAD K-A (dir.) (2005) Conflict and Governance. Springer Verlag, Heidelberg.

GRANDCLEMENT A (2016) Articuler approche relationnelle et approche spatiale des réseaux: application au cas des pôles de compétitivité. Géographie, Économie, Sociétés 18 (4) : 471-492.

Gumuchian H, Pecqueur B (2007) La Ressource Territoriale. Anthropos, Economica, Paris.

HatChuel A, WeIl B (1992) L'expert et le système. Gestion des savoirs et métamorphose des acteurs dans l'entreprise industrielle. Economica, Paris.

HiRSChMAN A-O (1970 et 1995) Défection et Prise de Parole. Fayard, Paris.

INNIS H-A (1930) The Fur Trade in Canada: an introduction to Canadian economic history. Yale University Pressn New Haven.

ISARD W (1956) Regional science, the concept of region and regional structure. Papers and Proceedings of the Regional Science Association 2(10): 13-26.

JACOBSEN N-B (2006) Industrial Symbiosis in Kalundborg, Denmark: a Quantitative Assessment of Economic and Environmental Aspects. Journal of Industrial Ecology 10: 239-255.

JeNNEQUIN H, MIOTTI L, MOUHOUd E-L (2017) Mesurer et anticiper la vulnérabilité des territoires face aux risques de délocalisation : une analyse à partir de données sectorielles pour la France. Économie et Statistique 497-498: 127-148.

JOYAL A (2008) Les APL au Brésil : une adaptation opportune des SPL à la française ? Géographie, Économie, Société 10 (2) : 185-197.

KLein J-L, LAVILLE J-L, MOUlaert F (eds.) (2014) L'Innovation Sociale. Éditions Érès, Toulouse.

KOOIMAN J (2000) Societal governance: levels, modes, and orders of social-political interaction. In: PIERRE J (ed) Debating Governance. Authority, steering and democracy. Oxford University Press.

KRUGMAN P, VenABLES A-J (1995) Globalization and the Inequality of Nations, The Quarterly Journal of Economics 110(4): 857-880.

KRUGMAN P (1991) Increasing returns and the economic geography. Journal of Political Economy 99(3): 483-499. 
LACOUR C (1996) La tectonique des territoires : d'une métaphore à une théorisation. In : PECQUEUR B. (éd.) Dynamiques territoriales et mutations économiques. L'Harmattan, Paris : 25-48.

LAFONTAINE D (2005) Le développement régional et territorial : un nouveau paradigme ? In : LAFONTAINE D, JEAN B (eds.) Territoires et fonctions. T1., Editions du GRIDEQ.

Lascoumes P, Le Galés P (2007) Sociologie de l'action publique. Collection 128, Armand Colin, Paris.

Le Gales P (2014) Gouvernance. In : Boussaguet L, JaCQuot S, Ravinet P (eds) Dictionnaire des Politiques Publiques. Presses de SciencesPo, Paris.

LEROUX I (2006) Gouvernance territoriale et jeux de négociation. Pour une grille d'analyse fondée sur le paradigme stratégique. Négociations $2: 83-98$.

LORETO V, SerVedio V-D-P, Strogatz S-H, TRIA F (2017) Dynamics on expanding spaces: modeling the emergence of novelties. In: DeGLI EsPOsti M, AltMANN E-G, PACHET F (eds.) Creativity and Universality in Language. Springer Verlag.

LUCAS R-E (1988) On the mechanics of economic development. Journal of Monetary Economics 22: 3-42.

LUNDVALL B-A, MASKELl P (2000) Nation states and economic development: from national systems of production to national systems of knowledge creation and learning. In: CLARK G-L, FELDMAN M, GertLer G (eds) The Oxford Handbook of Economic Geography. Oxford University Press, Oxford.

Leloup F, MOYART L, PeCQueur B (2005) La gouvernance territoriale comme nouveau mode de coordination territoriale? Géographie, Économie, Société 7 (4) : 321-332.

MARKUSEN A (1996) Sticky Places in Slippery Space: a Typology of Industrial Districts. Economic Geography 72(3): 294-314.

MARSHALL A (1919) Industry and Trade. Mac Millan. Londres.

MARTINAIS E (2015) Le conflit comme mode de participation. Les habitants contestataires de la politique de prévention des risques industriels. Participations 13 (3) : 89-117.

MELÉ P (dir.) (2013) Conflits de proximité et dynamiques urbaines. Presses Universitaires de Rennes.

MESSAOUDI D (2014) Les délocalisations industrielles dans les mutations des systèmes productifs français. Revue Géographique de l'Est 54 / 1-2 [en ligne] https://journals.openedition.org/rge/5153\#quotation

MÉNARD C (1997) Le pilotage des formes organisationnelles hybrides. Revue Économique 48 (3) : $741-750$.

Mira-Bonnardel S, Geneau I, Serrafero P (2012) Naissance d'un écosystème d'affaires. Entre stratégie délibérée et stratégie chemin faisant. Revue Française de Gestion 222 : 23-134.

MOINE A (2006) Le territoire comme un système complexe : un concept opératoire pour l'aménagement du territoire, L'Espace Géographique 35 (2) : 115-132.

Mounoub E-M (1992) Changement technique et division internationale du travail. Economica, Paris.

MuCHNIK J, DE SAINTE MARIE C (2010) Le temps des Syal. Editions Quae, Versailles.

NELSON R (1993) National innovation systems: a comparative analysis. Oxford University Press; Oxford.

NelSON R, WINTER S (1982) An Evolutionary Theory of Economic Change. Belknap Press/Harvard University Press, Cambridge.

NAlebuff B, Brandenburger A (1996) La Co-opétition, une révolution dans la manière de jouer concurrence et coopération. Village Mondial.

OCDE (2001) Innovative Clusters. Drivers of National Innovation Systems. OCDE, Paris.

Oldenburg R (1991) The Great Good Place. Marlowe \& Company, New York.

Ostrom E (1990) Governing the Commons. The Evolution of Institutions for Collective action. Cambridge, Cambridge University Press.

PASQUIER R (2012) Le pouvoir régional. Presses de SciencesPo, Paris.

PAsquier R, Simoulin V, WeibSteIn J (2007) La gouvernance territoriale. Pratiques, discours et théories. LGDJ, Paris.

Perroux F (1964) L'économie du XXe siècle. PuF, Paris, $3^{e}$ éd.

Pierre J (ed.) (2000) Debating Governance. Authority, steering and democracy. Oxford University Press.

PORTER M-E (1985) Competitive Advantage. The Free press, New York. 
PORTER M-E (2003) The economic performance of regions. Regional Studies 37(6-7): 549-579.

Rey-Valette H, Chia E, Mathé S, Michel l, Nougarèdes B, Soulard Ch, Maurel P, Jarrige F, Barbe E, GUIHENEUF P-Y (2014) Comment analyser la gouvernance territoriale? Mise à l'épreuve d'une grille de lecture. Géographie, Économie, Société 16 (1) : 65-89.

RIST G (2013) Le développement. Histoire d'une croyance occidentale. Presses de SciencesPo, Paris.

ROSENBERG N (1982) Inside the Black Box: Technology and Economics. Cambridge University Press.

SCHUMPETER J (1911) Théorie de l'évolution économique. Recherches sur le profit, le crédit, l'intérêt et le cycle de la conjoncture. Traduction française 1935, Dalloz, Paris.

SimOndON G (1958) Du mode d'existence des objets techniques. Aubier-Montaigne.

STÖHR W-B (1986) Regional Innovation Complexes. Papers in Regional Science 59: 29-44.

StORPER M (1997) The Regional World, Territorial Development in a Global Economy. The Guilford Press. SuBra Ph (2016) Géopolitique Locale. Collection U., Armand Colin, Paris.

TieBOUt C-M (1956) A pure theory of local expenditures. Journal of Political Economy 64(5): 416-424.

TORRE A (2015) Théorie du développement territorial. Géographie, Économie, Société 17 : 273-288.

Torre A, Beuret J-E (2012) Proximités territoriales. Collection Anthropos, Economica, Paris.

Torre A, Kirat Th, Melot R, Pham H-V (2016) Les conflits d'usage et de voisinage de l'espace. Bilan d'un programme de recherche pluridisciplinaire. L'Information Géographique 80 : 8-29.

TORRE A, TRAVERSAC J-B (eds) (2011) Territorial Governance. Local Development, Rural Areas and Agrofood Systems. Springer Verlag, Heidelberg \& New York.

VAN DE POEL I (2000) On the role of outsiders in technical development. Technology Analysis \& Strategic Management 12(3): 383-397.

VICENTE J (2016) Économie des Clusters. La Découverte, Paris.

VITRY Ch, CHIA E (2016) Contextualisation d'un instrument et apprentissages pour I'action collective. Management \& Avenir $83: 121-141$.

VON HIPPEL (1988) The Sources of Innovation. MIT Press, Cambridge, Massachusetts.

WATKINS M-H (1977) Staple theory revisited. Journal of Canadian Studies 12(5): 85-95.

WettenHALL R (2003) The rhetoric and reality of public - private partnerships. Public Organization Review 3(1): 77-107.

ZIMMERMANN J-B (2005) Entreprises et territoires : entre nomadisme et ancrage territorial. La Revue de I'Ires $47: 21-36$.

\section{Notes}

1 - Nous leur accordons une importance équivalente, contrairement à l'approche initiale où la loyauté est souvent introduite comme une option supplémentaire. 